\title{
THE DETERMINANT ON FLAT CONIC SURFACES WITH EXCISION OF DISKS
}

\author{
DAVID A. SHER
}

(Communicated by Michael Hitrik)

\begin{abstract}
Let $M$ be a surface with conical singularities, and consider a family of surfaces $M_{\epsilon}$ obtained from $M$ by removing disks of radius $\epsilon$ around a subset of the conical singularities. Such families arise naturally in the study of the moduli space of flat metrics on higher-genus surfaces with boundary. In particular, they have been used by Khuri to prove that the determinant of the Laplacian is not a proper map on this moduli space when the genus $p \geq 1$. Khuri's work is closely related to the isospectral compactness results of Osgood, Phillips, and Sarnak. Our main theorem is an asymptotic formula for the determinant of $M_{\epsilon}$ as $\epsilon$ approaches zero up to terms which vanish in the limit. The proof uses the determinant gluing formula of Burghelea, Friedlander, and Kappeler along with an observation of Wentworth on the asymptotics of Dirichlet-to-Neumann operators. We then apply this theorem to extend and sharpen the results of Khuri.
\end{abstract}

\section{INTRODUCTION}

Let $(M, g)$ be a compact connected surface of volume $V$ with finitely many isolated conical singularities $P_{i}$ of cross-section $\alpha_{i} S_{\theta}^{1}$. In particular, in a neigborhood of each $P_{i}$, we assume that $(M, g)$ is isometric to $(0,3 / 2)_{r} \times S_{\theta}^{1}$ with the metric

$$
d r^{2}+r^{2} \alpha_{i}^{2} d \theta^{2}
$$

for some constant $\alpha_{i}>0$. We say that $2 \pi \alpha_{i}$ are the cone angles; note that when $\alpha_{i}=1$, the conic singularity at $P_{i}$ becomes smooth.

Choose a nonempty subset $\left\{P_{1}, P_{2}, \ldots, P_{k}\right\}$ of the conical singularities. For each $i$ with $1 \leq i \leq k$, fix a real number $b_{i} \in(0,1]$. For each $\epsilon \in(0,1]$, let $\Gamma_{\epsilon, i}$ be the curve $r=b_{i} \epsilon$, and let $\Gamma_{\epsilon}$ be the union of all $\Gamma_{\epsilon, i}$. The region inside $\Gamma_{\epsilon, i}$ is a cone of angle $2 \pi \alpha_{i}$ and length $b_{i} \epsilon$, which we call $C_{\alpha_{i}, b_{i} \epsilon}$. We then let $M_{\epsilon}$ be the connected manifold with boundary obtained by removing all of the $C_{\alpha_{i}, b_{i} \epsilon}$ from $M$; its boundary is precisely $\Gamma_{\epsilon}$. Finally, let

$$
\beta=\frac{1}{k} \sum_{j=1}^{k} b_{1} \ldots \hat{b}_{j} \ldots b_{k},
$$

where the hat symbol indicates that the $j$ th factor is omitted from the product.

Our main theorem is the following asymptotic expansion.

Received by the editors February 25, 2013 and, in revised form, July 8, 2013.

2010 Mathematics Subject Classification. Primary 58J50, 58J52.

The author was partially supported by an ARCS fellowship in 2011-2012 and a CRM-ISM fellowship in 2012-2013. 
Theorem 1.1. Let $M_{\epsilon}$ be as defined above. As $\epsilon \rightarrow 0$, we have

$$
\log \operatorname{det} \Delta_{M_{\epsilon}}=\left(k-1+\sum_{i=1}^{k} \frac{1}{6}\left(\alpha_{i}+\frac{1}{\alpha_{i}}\right)\right) \log \epsilon+(k-1) \log \log (1 / \epsilon)+\log \operatorname{det} \Delta_{M}
$$

(1.1) $-\sum_{i=1}^{k} \log \operatorname{det} \Delta_{C_{\alpha_{i}, 1}}+k \log 2-\log V+\sum_{i=1}^{k} \frac{1}{6}\left(\alpha_{i}+\frac{1}{\alpha_{i}}\right) \log b_{i}+\log \beta+o(1)$.

Here we are imposing Dirichlet boundary conditions at all boundaries and the Friedrichs extension at all conic singularities. We also ignore the zero eigenvalue of $\Delta_{M}$ for the purposes of defining its determinant.

Each term in the expansion except $\log \operatorname{det} \Delta_{M}, \log \operatorname{det} \Delta_{M_{\epsilon}}$, and $\log V$ may be computed explicitly as a function of the cone angles $\alpha_{i}$ and the constants $b_{i}$. In particular, Spreafico has computed a formula for $\log \operatorname{det} \Delta_{C_{\alpha, 1}}[\mathrm{Sp}$. The error is only $o(1)$ in general, but in the $k=1$ case there is an explicit upper bound:

Theorem 1.2. When $k=1, b_{1}=1$, and $\epsilon \leq 2^{-\alpha_{1}}$, the error in (1.1) is bounded by $12 \epsilon^{1 / \alpha_{1}}$.

1.1. Relation to isospectral compactness. In a series of papers OPS1, OPS2, OPS3 from the 1980s, Osgood, Phillips, and Sarnak investigate sets of isospectral metrics on surfaces. Their key result is that in the setting of closed surfaces, any set of isospectral metrics is compact in the natural $C^{\infty}$ topology. The proof makes extensive use of the determinant of the Laplacian. First, in OPS1, Osgood, Phillips, and Sarnak prove that within any conformal class of metrics (normalized so that the area is constant) on a closed surface, the determinant of the Laplacian is maximized by the constant curvature metric. They then show that on the moduli space of constant curvature metrics, the log-determinant of the Laplacian is a proper map. These two facts are key to the proof of isospectral compactness. In OPS3, an analogous result is proved for isospectral sets of planar domains (normalized so that the boundary length is constant), with the usual flat Euclidean metric and Dirichlet boundary conditions.

Khuri, in Kh, Kh2, addressed the natural question of whether a similar result holds for isospectral sets of flat metrics on a topological surface of genus $p$ with $n$ disks removed, denoted $\Sigma_{p, n}$. The setting $p=0$ is precisely the case of planar domains and $n=0$ is the case of closed surfaces, so Khuri only considered $n p \geq 1$. For $n p \geq 1$, Khuri showed by constructing counterexamples that the log-determinant is not a proper map on the appropriate moduli space of flat metrics Kh2]. The counterexamples are closely related to our construction of $M_{\epsilon}$; namely, Khuri took surfaces $(M, g)$ of genus $p$ with $n$ isolated conic singularities and removed a disk of radius $\epsilon$ around each singular point, then let $\epsilon$ go to zero. After normalizing so that the boundary length is constant, Khuri showed that the resulting surfaces $\left(M_{\epsilon}, \hat{g}\right)$ approach the boundary of moduli space but have $\log \operatorname{det} \Delta_{\left(M_{\epsilon}, \hat{g}\right)}$ bounded below. Therefore, the Osgood-Phillips-Sarnak approach does not work for $n p \geq 1 \mathrm{Kh} 2$. On the other hand, using a comparison of two different moduli spaces, Y.-H. Kim has recently proved isospectral compactness of sets of flat metrics on $\Sigma_{p, n}$ in this $n p \geq 1$ setting $\mathrm{Kim}$. The problem remains open for all $p$ and all $n \geq 1$ if the isospectral metrics are not assumed to be flat.

Our goal is to study the behavior of the determinant of the Laplacian as a function on the space of metrics on $\Sigma_{p, n}$. Based on the results of Khuri and Kim, 
we expect that this work may have further applications to the isospectral problem and related questions. As a first step, we apply Theorem 1.1 to sharpen the results of Khuri in $\mathrm{Kh}, \mathrm{Kh} 2$. Let $(M, g)$ be a fixed surface of genus $p$, where $g$ is a flat conical metric with $n$ conical singularities, and let $V$ be the volume of $M$ as before. Let $M_{\epsilon}$ be the surface obtained from $M$ by removing a cone of radius $\epsilon$ around each of the $n$ cone points. We can then apply Theorem 1.1 to $M_{\epsilon}$, with $k=n$ and $b_{i}=1$ for all $i$. However, Khuri normalizes so that the geodesic curvature is constant and equal to -1 on the boundary, which means multiplying the metric on $M_{\epsilon}$ by $\epsilon^{-2}$; let $\hat{g}=\epsilon^{-2} g$. By Proposition 2.3.

$$
\log \operatorname{det} \Delta_{\left(M_{\epsilon}, \hat{g}\right)}=\log \operatorname{det} \Delta_{\left(M_{\epsilon}, g\right)}+2 \zeta_{M_{\epsilon}}(0) \log \epsilon .
$$

On the other hand, since $M_{\epsilon}$ is a smooth surface with boundary, the McKean-Singer heat asymptotics of [MS] (a good exposition may also be found in [Ros]) imply that

$$
\zeta_{M_{\epsilon}}(0)=\frac{1}{6} \chi\left(M_{\epsilon}\right)=\frac{1}{6}(\chi(M)-n)=\frac{1}{6}(2-2 p-n) .
$$

Moreover, by the Gauss-Bonnet theorem, the sum of all $\alpha_{i}$ must be equal to $2 p+n-2$ Kh]. Combining Theorem 1.1 with (2.3), (1.3), and the Gauss-Bonnet theorem, we have shown:

Proposition 1.3. The log-determinant of the Laplacian of Khuri's metrics $\left(M_{\epsilon}, \hat{g}\right)$ has an expansion as $\epsilon \rightarrow 0$ :

$$
\begin{gathered}
\log \operatorname{det} \Delta_{\left(M_{\epsilon}, \hat{g}\right)}=\frac{1}{6}\left(2-2 p-n+\sum_{i=1}^{n}\left(\frac{1}{\alpha_{i}}\right)\right) \log \epsilon+(n-1) \log \log 1 / \epsilon \\
+\log \operatorname{det} \Delta_{(M, g)}-\sum_{i=1}^{n} \log \operatorname{det} \Delta_{C_{\alpha_{i}, 1}}+n \log 2-\log V+o(1) .
\end{gathered}
$$

In the $n=1$ case, we also obtain a better error estimate from Theorem 1.2 As a consequence of Proposition 1.3, we conclude that

Proposition 1.4. The log-determinant $\log \operatorname{det} \Delta_{\left(M_{\epsilon}, \hat{g}\right)}$ approaches $-\infty$ as $\epsilon \rightarrow 0$ iff

$$
\sum_{i=1}^{n} \frac{1}{\alpha_{i}}>2 p+n-2 .
$$

Notice that as any individual $\alpha_{i}$ goes to zero, condition (1.5) is eventually satisfied; in particular, if even one of the cone angles is small enough, the log-determinant of $M_{\epsilon}$ will go to $-\infty$ as $\epsilon \rightarrow 0$. This observation suggests that it might be fruitful to analyze the behavior of the expansion in Proposition 1.3 as one or more of the cone angles $\alpha_{i}$ degenerate to zero; the reward would be a much better understanding of the determinant on moduli space. Such an analysis would likely require a joint asymptotic expansion in $\epsilon$ and $\alpha_{i}$, which seems beyond the reach of the methods we use here. Various microlocal techniques for analysis on manifolds with conic singularities, such as those discussed in [Mo or in [GKM], could prove useful.

1.2. Examples. We now examine some particular cases. First we let $p=1$ and $n=1$; in this setting, Khuri proved that $\log \operatorname{det} \Delta_{\left(M_{\epsilon}, \hat{g}\right)}$ is bounded below (equation 4.3 .15 of $[\mathrm{Kh}])$. Note that by the Gauss-Bonnet theorem, the only cone angle $\alpha$ must be 1. Plugging $p=1, n=1$, and $\alpha=1$ into (1.4), we see that the coefficients 
of $\log \epsilon$ and $\log \log 1 / \epsilon$ vanish, and hence the log-determinants actually converge to a constant. This sharpens Khuri's result.

Next we let $p=1$ and $n \geq 2$. In this case, Khuri showed (equation 4.3 .19 of $\underline{\mathrm{Kh}}$ ) that if all cone angles $\alpha_{i}$ are equal to 1 ,

$$
\log \operatorname{det} \Delta_{\left(M_{\epsilon}, \hat{g}\right)} \geq(n-1) \log \log 1 / \epsilon+C .
$$

The coefficient of $\log \epsilon$ in our formula again vanishes, and the coefficient of $\log \log 1 / \epsilon$ is precisely $n-1$, which shows that Khuri's inequality is sharp and also identifies the constant $C$. On the other hand, when the cone angles $\alpha_{i}$ are not all equal to 1 , they must still sum to $n$, so $\sum_{i=1}^{n} \alpha_{i}^{-1}>n$, and the coefficient of $\log \epsilon$ is positive. In this case, the log-determinants do in fact decrease to $-\infty$ as $\epsilon \rightarrow 0$; Khuri did not consider this case.

Finally, we let $p \geq 2$ and $n=1$. Here the cone angle $\alpha_{i}$ must be $2 p-1$. The coefficient of the $\log |\log \epsilon|$ term vanishes, but the coefficient of $\log \epsilon$ is precisely

$$
\frac{1}{6}\left(1-2 p+\frac{1}{2 p-1}\right) .
$$

When $p \geq 2$, this is negative, so the log-determinants increase to $\infty$ as $\epsilon \rightarrow 0$. It must be noted that the leading-order coefficient of $\log \epsilon$ is inconsistent with equation 4.3.22 in [Kh]. However, the calculation in $\mathrm{Kh}$ is not correct, as the asymptotic behavior of the determinant of an annulus is computed with the Euclidean metric when it should be computed with the conic metric.

1.3. Organization. The proof of Theorems 1.1 and 1.2 proceeds in two steps. First we use the gluing formula of Burghelea, Friedlander, and Kappeler BFK] to break up the manifold $M$ along $\Gamma_{\epsilon}$ and decompose $\log \operatorname{det} \Delta_{M}$ as a sum of the log-determinants of the individual pieces. The only nonexplicit term in the BFK formula is the log-determinant of the Neumann jump operator on $\Gamma_{\epsilon}$. In the second step, we compute the asymptotics of this Neumann jump operator as $\epsilon \rightarrow 0$. This computation is based closely on an observation of Wentworth We. The combination of Wentworth's observation and the BFK gluing formula is a natural one which has proven useful in other investigations of the determinant on moduli space $[\mathrm{Ko}$.

\section{Determinant GLuing Formula}

In this section, we recall the Burghelea-Friedlander-Kappeler gluing formula and apply it to our problem. For any $\epsilon>0$, we define an operator $R_{\epsilon}$ on $\Gamma_{\epsilon}$, following [BFK]: if $f$ is a function on $\Gamma_{\epsilon}$, we let $u_{+}$be the solution of $\Delta_{M_{\epsilon}} u_{+}=0$ with $\left.u_{+}\right|_{\Gamma_{\epsilon}}=f$. Similarly, let $u_{-}$be the harmonic function on the union of the $C_{\alpha_{i}, b_{i} \epsilon}$ with boundary data $f$. As always, we require $u_{-}$and $u_{+}$to be in the Friedrichs domain at all conic singularities. Then in a neighborhood of $\Gamma_{\epsilon}, g=\partial_{r} u_{-}-\partial_{r} u_{+}$ is well-defined; we let $R_{\epsilon} f$ be the restriction of $g$ to $\Gamma_{\epsilon}$. In fact, $R_{\epsilon}$ is simply the sum of the Dirichlet-to-Neumann operators on $M_{\epsilon}$ and $C_{\alpha_{i}, b_{i} \epsilon}$, and we call it the Neumann jump operator for $\Gamma_{\epsilon}$.

It is well-known that $R_{\epsilon}$ is an elliptic pseudodifferential operator of order 1 and that it is possible to define a zeta function and determinant of $R_{\epsilon}$ in the usual fashion $\mathrm{BFK}$. In this paper, when we define determinants, we always leave out the zero eigenvalues. For $R_{\epsilon}$, there is precisely one. 
Lemma 2.1. The kernel of $R_{\epsilon}$ is equal to the set of globally constant functions on $\Gamma_{\epsilon}$.

Proof. Suppose that $f$ is in the kernel of $R_{\epsilon}$, and construct $u_{+}$and $u_{-}$as above. Since $\partial_{r} u_{-}=\partial_{r} u_{+}$on $\Gamma_{\epsilon}$ and $u_{+}=u_{-}$on $\Gamma_{\epsilon}, u_{+}$and $u_{-}$glue together to define a $C^{1}$ function $u$ on $M$ which solves $\Delta_{M} u=0$ weakly. By elliptic regularity, $u$ is in fact a smooth harmonic function on $M$ away from the cone points. Near each cone point $P_{i}$, let $r_{i}$ be the radial coordinate. Since $u$ is in the Friedrichs domain, $u$ is bounded near each cone point. Moreover, using the explicit form of harmonic functions on an exact cone (see (3.7) below), there is a constant $C$ such that $|\nabla u| \leq C r_{i}^{-1+1 / \alpha_{i}}$ near each cone point. These bounds justify the integration by parts which leads to

$$
\int_{M} u \Delta_{M} u=\int_{M}|\nabla u|^{2}
$$

therefore, since $u$ is harmonic, $u$ must be constant.

We now have the following gluing formula, due to Burghelea, Friedlander, and Kappeler [BFK] in the smooth setting and to Loya, McDonald and Park [LMP] in the setting of manifolds with conical singularities:

Proposition 2.2. BFK

$\log \operatorname{det} \Delta_{M}=\log \operatorname{det} \Delta_{M_{\epsilon}}+\sum_{i=1}^{k} \log \operatorname{det} \Delta_{C_{\alpha_{i}, b_{i} \epsilon}}+\log V-\sum_{i=1}^{k} \log 2 \pi \alpha_{i} b_{i} \epsilon+\log \operatorname{det} R_{\epsilon}$.

Note that the length of $\Gamma_{\epsilon}$ is exactly $\sum_{i=1}^{k} 2 \pi \alpha_{i} b_{i} \epsilon$. Rearranging (2.1) gives us a formula for $\log \operatorname{det} \Delta_{M_{\epsilon}}$ in terms of the other undetermined quantities:

$$
\begin{aligned}
\log \operatorname{det} \Delta_{M_{\epsilon}}= & \log \operatorname{det} \Delta_{M}-\sum_{i=1}^{k} \log \operatorname{det} \Delta_{C_{\alpha_{i}, b_{i} \epsilon}}-\log V \\
& +\sum_{i=1}^{k} \log 2 \pi \alpha_{i} b_{i}+k \log \epsilon-\log \operatorname{det} R_{\epsilon} .
\end{aligned}
$$

To simplify the formula, we make a scaling observation:

Proposition 2.3. Let $(\Omega, g)$ be any compact Riemannian surface, with or without boundary and with or without isolated conic singularities. Then

$$
\log \operatorname{det} \Delta_{\Omega, \epsilon^{2} g}=\log \operatorname{det} \Delta_{\Omega, g}-2 \zeta_{\Omega, g}(0) \log \epsilon .
$$

Proof. If we scale the metric $g$ by $\epsilon^{2}$, we scale the eigenvalues of the Laplacian by $\epsilon^{-2}$. The proposition then follows from taking the derivative of $\epsilon^{2 s} \zeta_{\Omega, g}(0)$ at $s=0$.

Note that, crucially, $\zeta_{\Omega, g}(s)$ has no pole at $s=0$; this is not true for manifolds with conic singularities in general, but is true in two dimensions, where the crosssection is a circle (see [Kh2 for a detailed explanation). If a pole were present, an analogue of Proposition 2.3 would still hold (using the Laurent series definition of the determinant), but with extra terms. 
The special value $\zeta_{\Omega, g}(0)$ is known in many cases. In particular, a result of Spreafico $[\mathrm{Sp}$ states that

$$
\zeta_{C_{\alpha, 1}}(0)=\frac{1}{12}\left(\alpha+\frac{1}{\alpha}\right) .
$$

Combining this with (2.2), using Proposition 2.3 and rearranging, we obtain

$$
\begin{gathered}
\log \operatorname{det} \Delta_{M_{\epsilon}}=\left(k+\sum_{i=1}^{k} \frac{1}{6}\left(\alpha_{i}+\frac{1}{\alpha_{i}}\right)\right) \log \epsilon+\log \operatorname{det} \Delta_{M}-\sum_{i=1}^{k} \log \operatorname{det} \Delta_{C_{\alpha_{i}, 1}} \\
+\sum_{i=1}^{k} \frac{1}{6}\left(\alpha_{i}+\frac{1}{\alpha_{i}}\right) \log b_{i}+\sum_{i=1}^{k} \log 2 \pi \alpha_{i} b_{i}-\log \operatorname{det} R_{\epsilon}-\log V .
\end{gathered}
$$

\section{The Neumann Jump operator}

We now prove the following asymptotic expansion for $\log \operatorname{det} R_{\epsilon}$ as $\epsilon$ goes to zero; combining it with (2.3) yields Theorem 1.1 .

Theorem 3.1. As $\epsilon \rightarrow 0$,

$$
\log \operatorname{det} R_{\epsilon}=\log \epsilon-(k-1) \log \log (1 / \epsilon)+\sum_{i=1}^{k} \log \pi b_{i} \alpha_{i}-\log \beta+o(1) .
$$

Moreover, when $k=1, b_{1}=1$, and $\epsilon \leq 2^{-\alpha_{1}}$, the error in (3.1) is bounded by $12 \epsilon^{1 / \alpha_{1}}$.

The proof of this theorem in the special case $k=1, \alpha_{1}=1$, and $b_{1}=1$ is due to Wentworth [We, albeit without the error bound; we adapt the proof given there.

3.1. Computation of $R_{\epsilon}$. Identifying $\Gamma_{\epsilon}$ with $\oplus_{i=1}^{k} S^{1}$, we view $R_{\epsilon}$ as an operator on $L^{2}\left(\oplus_{i=1}^{k} S^{1}\right)$. The space $L^{2}\left(\oplus_{i=1}^{k} S^{1}\right)$ admits an orthogonal decomposition

$$
L^{2}\left(\oplus_{i=1}^{k} S^{1}\right)=\mathcal{K} \oplus \mathcal{C} \oplus L_{0}^{2} .
$$

Here $\mathcal{K}$ is the one-dimensional space of globally constant functions, $\mathcal{C}$ is the $k-1$ dimensional space of locally constant functions which integrate to zero, and $L_{0}^{2}$ is the subspace of $L^{2}\left(\oplus_{i=1}^{k} S^{1}\right)$ spanned by the nonconstant eigenfunctions on each component. Let $\Pi_{\mathcal{K}}, \Pi_{\mathcal{C}}$, and $\Pi_{0}$ be the orthogonal projections onto each summand in (3.2). As we have observed, the kernel of $R_{\epsilon}$ is precisely $\mathcal{K}$. Moreover, by Green's theorem, the image of $R_{\epsilon}$ is orthogonal to $\mathcal{K}$.

We define an operator $L_{\epsilon}$ on $C^{\infty}\left(\oplus_{i=1}^{k} S^{1}\right)$ as follows: for any smooth function $f$ on $\oplus_{i=1}^{k}\left(S^{1}\right)$, let $u_{+}$be the harmonic function on $M_{\epsilon}$ with boundary values $f$ on $\Gamma_{\epsilon}$. Then let $L_{\epsilon}(f)=\left.\left(u_{+}\right)\right|_{\Gamma_{1}}$. Note that $L_{\epsilon}$ is essentially the inverse of the operator $\mathcal{E}_{R(\epsilon)}$ from [We; we use $L_{\epsilon}$ instead to avoid issues with the domain of the extension operator $\mathcal{E}_{R(\epsilon)}$. We also observe that $L_{\epsilon}$ is the identity operator on $\mathcal{K}$. The following proposition is key:

Proposition 3.2. For each $\epsilon<1$ and each $f \in \mathcal{C} \oplus L_{0}^{2}$, we also have $L_{\epsilon} f \in \mathcal{C} \oplus L_{0}^{2}$. Moreover, for any $f \in C^{\infty}\left(\oplus_{i=1}^{k} S^{1}\right)$ and any $\epsilon<1$,

$$
\left\|L_{\epsilon} f\right\|_{L^{2}\left(\oplus_{i=1}^{k} S^{1}\right)} \leq\|f\|_{L^{2}\left(\oplus_{i=1}^{k} S^{1}\right)} .
$$

Note that the second statement allows us to extend $L_{\epsilon}$ by continuity to an operator on $L^{2}\left(\oplus_{i=1}^{k} S^{1}\right)$ which is bounded in operator norm by 1 . We call this extension $L_{\epsilon}$ as well. 
Proof. The proofs are identical to the proofs of Lemmas 3.3 and 3.4 in [We] and involve integration and Green's theorem; we will not repeat them here.

For each nonzero $n \in \mathbb{Z}$ and $j$ between 1 and $k$, let $f_{n, j}$ be the function on $\oplus_{i=1}^{k} S^{1}$ which is equal to $e^{i n \theta}$ on the $j$ th component of $S^{1}$ and zero on all the other components. The collection of $f_{n, j}$ forms a basis of $L_{0}^{2}$. Again following We and using the same notation, we define auxiliary operators $T_{\epsilon}, U_{\epsilon}^{ \pm}, \mathcal{V},|\mathcal{V}|$, and $B$ on $L_{0}^{2}$ by

$$
\begin{array}{cc}
T_{\epsilon} f_{n, j}=\frac{\epsilon^{n / \alpha_{j}}-\epsilon^{-n / \alpha_{j}}}{\epsilon^{n / \alpha_{j}}+\epsilon^{-n / \alpha_{j}}} f_{n, j}, & U_{\epsilon}^{ \pm} f_{n, j}=\frac{1}{2}\left(\epsilon^{n / \alpha_{j}} \pm \epsilon^{-n / \alpha_{j}}\right) f_{n, j}, \\
\mathcal{V} f_{n, j}=\frac{n}{\alpha_{j}} f_{n, j}, \quad|\mathcal{V}| f_{n, j}=\frac{|n|}{\alpha_{j}} f_{n, j},
\end{array}
$$

extending by linearity to $L_{0}^{2}$. Note that each of these operators is invertible on $L_{0}^{2}$; the inverses may be written explicitly. Additionally, let $f_{0, j}$ be the function which is 1 on the $j$ th component of $S^{1}$ and zero on the others; these form a basis for $\mathcal{K} \oplus \mathcal{C}$. Finally, let $\bar{B}$ be the multiplication operator on $L^{2}\left(\oplus_{i=1}^{k} S^{1}\right)$ with $\bar{B} f_{n, j}=b_{j} f_{n, j}$ for both zero and nonzero $n$; it restricts to an operator on $L_{0}^{2}$ but not to an operator on $\mathcal{C}$, so write $B=\left(\Pi_{0}+\Pi_{\mathcal{C}}\right) \bar{B}\left(\Pi_{0}+\Pi_{\mathcal{C}}\right)$.

We now compute $R_{\epsilon}$ on $\mathcal{C} \oplus L_{0}^{2}$ in terms of these auxiliary operators. Let $\mathcal{D}_{M_{\epsilon}}$ be the Dirichlet-to-Neumann operator for $M_{\epsilon}$ and $\mathcal{D}_{C_{\epsilon}}$ be the Dirichlet-to-Neumann operator for $\oplus_{i=1}^{k} C_{\alpha_{i}, b_{i} \epsilon}$; then $R_{\epsilon}=\mathcal{D}_{M_{\epsilon}}+\mathcal{D}_{C_{\epsilon}}$. As before, by Stokes' theorem, each of these operators also preserves the orthogonal decomposition. Moreover, we may compute $\mathcal{D}_{C_{\epsilon}}$ directly. Suppose that the boundary data is $f_{m, j}$. Then by separation of variables, the harmonic extension to $C_{\alpha_{j}, b_{j} \epsilon}$ is precisely $\left(r / b_{j} \epsilon\right)^{|m| / \alpha_{j}} e^{i m \theta}$, and the harmonic extensions on the other components are zero. The inward-pointing normal derivative at the boundary is $-\partial_{r}$, so we conclude that

$$
\mathcal{D}_{C_{\epsilon}} f_{m, j}=-\frac{|m|}{b_{j} \epsilon \alpha_{j}} f_{m, j}=-\frac{1}{b_{j} \epsilon}|\mathcal{V}| f_{m, j} .
$$

By linearity, we see that $\epsilon \mathcal{D}_{C_{\epsilon}}=-B^{-1}|\mathcal{V}|$ on $L_{0}^{2}$; note that $\mathcal{D}_{C_{\epsilon}}=0$ on $\mathcal{C}$, since the harmonic extension of a constant function on $\Gamma_{\epsilon, i}$ to $C_{\alpha_{i}, b_{i} \epsilon}$ which is in the Friedrichs domain is itself constant. It remains to analyze $\mathcal{D}_{M_{\epsilon}}$.

Lemma 3.3. We have the following four equations for $\epsilon \mathcal{D}_{M_{\epsilon}}$ :

$$
\begin{gathered}
\Pi_{0}\left(\epsilon \mathcal{D}_{M_{\epsilon}}\right) \Pi_{0}=B^{-1} \Pi_{0}\left(\mathcal{V}\left(\mathcal{T}_{\epsilon}\right)^{-1}-\mathcal{V}\left(\mathcal{U}_{\epsilon}^{-}\right)^{-1} L_{\epsilon}\right) \Pi_{0}, \\
\Pi_{\mathcal{C}}\left(\epsilon \mathcal{D}_{M_{\epsilon}}\right) \Pi_{0}=B^{-1} \Pi_{\mathcal{C}}\left(\frac{1}{\log (1 / \epsilon)} L_{\epsilon}\right) \Pi_{0}, \\
\Pi_{0}\left(\epsilon \mathcal{D}_{M_{\epsilon}}\right) \Pi_{\mathcal{C}}=B^{-1} \Pi_{0}\left(-\mathcal{V}\left(\mathcal{U}_{\epsilon}^{-}\right)^{-1} L_{\epsilon}\right) \Pi_{\mathcal{C}}, \\
\Pi_{\mathcal{C}}\left(\epsilon \mathcal{D}_{M_{\epsilon}}\right) \Pi_{\mathcal{C}}=B^{-1} \Pi_{\mathcal{C}}\left(\frac{1}{\log (1 / \epsilon)}\left(L_{\epsilon}-I d\right)\right) \Pi_{\mathcal{C}}
\end{gathered}
$$

Proof. First we prove (3.3) and (3.4). It is enough to prove the statements for the action of the operators on each $f_{m, j}$ for nonzero $m$. Let $u_{+}$be the solution of the Dirichlet problem on $M_{\epsilon}$ with boundary data equal to $f_{m, j}$. Since $u_{+}$is harmonic, 
we may write that in a radius 3/2-neighborhood of each conic point, where $r_{i}$ is the radial coordinate near $P_{i}$,

$u_{+}(r, \theta)=\sum_{n \neq 0,1 \leq i \leq k}\left(a_{n, i}^{+}\left(\frac{r_{i}}{b_{i} \epsilon}\right)^{n / \alpha_{i}}+a_{n, i}^{-}\left(\frac{r_{i}}{b_{i} \epsilon}\right)^{-n / \alpha_{i}}\right) f_{n, i}+\sum_{i=1}^{k}\left(a_{0, i}^{+}+a_{0, i}^{-} \log \frac{r_{i}}{b_{i} \epsilon}\right) f_{0, i}$.

Since $u_{+}\left(b_{i} \epsilon, \theta\right)=f_{m, j}$, we see that $a_{n, i}^{+}+a_{n, i}^{-}=\delta_{(n, i),(m, j)}$ for nonzero $m$ and that $a_{0, i}^{+}=0$, for each $i$. Therefore $u_{+}$may be rewritten

$u_{+}(r, \theta)=\left(\frac{r_{j}}{b_{j} \epsilon}\right)^{-m / \alpha_{j}} f_{m, j}+\sum_{n \neq 0,1 \leq i \leq k} a_{n, i}^{+}\left(\left(\frac{r_{i}}{b_{i} \epsilon}\right)^{n / \alpha_{i}}-\left(\frac{r_{i}}{b_{i} \epsilon}\right)^{-n / \alpha_{i}}\right) f_{n, i}+\sum_{i=1}^{k} a_{0, i}^{-} \log \frac{r_{i}}{b_{i} \epsilon} f_{0, i}$.

Now $\mathcal{D}_{M_{\epsilon}} f_{m, j}=\left.\left(\partial_{r_{i}}\left(u_{+}\right)\right)\right|_{r_{i}=b_{i} \epsilon}$ and $L_{\epsilon} f_{m, j}=\left.u_{+}\right|_{r_{i}=b_{i}}$. Since the other operators in (3.3) are multiplication operators on each mode and the projection operators single out the components $f_{m, j}$ with nonzero $m$, it is easy to compute both sides of (3.3) in terms of the $a_{n, i}^{+}$. We find that both sides of (3.3) are equal to

$$
-\frac{m}{\alpha_{j} b_{j}} f_{m, j}+\sum_{n \neq 0,1 \leq i \leq k} \frac{2 n}{\alpha_{i} b_{i}} a_{n, i}^{+} f_{n, i} .
$$

This completes the proof of (3.3). Similarly, from (3.8), we compute that both sides of (3.4) are equal to

$$
\sum_{i=1}^{k} a_{0, i}^{-} \frac{1}{b_{i}} f_{0, i}
$$

which verifies (3.4).

On the other hand, to prove (3.5) and (3.6), it is enough to prove the statements for the action of the operators on each $f_{0, j}$. As before, let $u_{+}$be the solution of the Dirichlet problem on $M_{\epsilon}$ with boundary data $f_{0, j}$. Then we may still write (3.7), and the boundary conditions allow us to simplify to

$$
u_{+}(r, \theta)=f_{0, j}+\sum_{n \neq 0,1 \leq i \leq k} a_{n, i}^{+}\left(\left(\frac{r_{i}}{b_{i} \epsilon}\right)^{n / \alpha_{i}}-\left(\frac{r_{i}}{b_{i} \epsilon}\right)^{-n / \alpha_{i}}\right) f_{n, i}+\sum_{i=1}^{k} a_{0, i}^{-} \log \frac{r_{i}}{b_{i} \epsilon} f_{0, i} .
$$

We may then compute, as before, that both sides of (3.5) are equal to

$$
\sum_{n \neq 0,1 \leq i \leq k} \frac{2 n}{\alpha_{i} b_{i}} a_{n, i}^{+} f_{n, i}
$$

and both sides of (3.6) are

$$
\sum_{i=1}^{k} a_{0, i}^{-} \frac{1}{b_{i}} f_{0, i} .
$$

This completes the proof of the lemma.

Combining the lemma with the preceding remarks on $\mathcal{D}_{C_{\epsilon}}$ gives a formula for $\epsilon R_{\epsilon}$ in terms of $L_{\epsilon}$ and the auxiliary operators; see Proposition 3.4 below. 
3.2. Determinant asymptotics. In order to compute the asymptotics of the determinant of $\epsilon R_{\epsilon}$, we view $\epsilon R_{\epsilon}$ as the sum of a diagonal operator $A_{\epsilon}$ and a 'small perturbation' $K_{\epsilon}$. The following proposition is immediate from the previous lemma and discussion:

Proposition 3.4. Let $A_{\epsilon}$ and $K_{\epsilon}$ be operators on $\mathcal{C} \oplus L_{0}^{2}$ given by

Then $\epsilon R_{\epsilon}=A_{\epsilon}+K_{\epsilon}$.

$$
\begin{gathered}
B A_{\epsilon}=-2 \Pi_{0}|\mathcal{V}| \Pi_{0}-\Pi_{\mathcal{C}} \frac{1}{\log (1 / \epsilon)} \Pi_{\mathcal{C}} \\
B K_{\epsilon}=\Pi_{0}\left(|\mathcal{V}|+\mathcal{V}\left(\mathcal{T}_{\epsilon}\right)^{-1}-\mathcal{V}\left(\mathcal{U}_{\epsilon}^{-}\right)^{-1} L_{\epsilon}\right) \Pi_{0}+\Pi_{\mathcal{C}}\left(\frac{1}{\log (1 / \epsilon)} L_{\epsilon}\right) \Pi_{0} \\
+\Pi_{0}\left(-\mathcal{V}\left(\mathcal{U}_{\epsilon}^{-}\right)^{-1} L_{\epsilon}\right) \Pi_{\mathcal{C}}+\Pi_{\mathcal{C}}\left(\frac{1}{\log (1 / \epsilon)} L_{\epsilon}\right) \Pi_{\mathcal{C}}
\end{gathered}
$$

The key to the proof of Theorem 3.1 is that the asymptotics of the determinant of $\epsilon R_{\epsilon}$ are closely related to those of the determinant of $A_{\epsilon}$, which are easy to compute. The following lemma, whose proof is deferred until the next section, provides the necessary comparison:

Lemma 3.5. As $\epsilon \rightarrow 0$,

$$
\log \operatorname{det} \epsilon R_{\epsilon}-\log \operatorname{det} A_{\epsilon}=o(1) .
$$

Moreover, when $k=1, b_{1}=1$, and $\epsilon \leq 2^{-\alpha_{1}}$,

$$
\left|\log \operatorname{det} \epsilon R_{\epsilon}-\log \operatorname{det} A_{\epsilon}\right| \leq 12 \epsilon^{1 / \alpha_{1}} \text {. }
$$

We now compute the asymptotics of the determinant of $A_{\epsilon}$. The eigenvalues of $2 B^{-1}|\mathcal{V}|$ on $L_{0}^{2}$ are precisely $2 m /\left(b_{i} \alpha_{i}\right)$ for each $m$ in $\mathbb{N}$ and each $i$ between 1 and $k$, each with multiplicity 2 . The zeta function for $2|\mathcal{V}|$ is therefore

$$
\sum_{i=1}^{k} 2\left(\frac{2}{b_{i} \alpha_{i}}\right)^{-s} \zeta_{\text {Riem }}(s) \text {. }
$$

Taking the derivative at zero and using the special values of the Riemann zeta function, we compute that the log-determinant of $A_{\epsilon}$ on $L_{0}^{2}$ is $\sum_{i=1}^{k} \log \pi b_{i} \alpha_{i}$. Moreover, to compute the log-determinant of $A_{\epsilon}$ on $\mathcal{C}$, first let $\hat{B}$ be the $(k-1)$-dimensional matrix $\Pi_{C} B \Pi_{C}$; it is easy to see that $\hat{B}$ is invertible with all positive eigenvalues. Then the log-determinant of $A_{\epsilon}$ on $\mathcal{C}$ (note that we take absolute values of the eigenvalues first) is just

$\log \operatorname{det} \hat{B}^{-1}+(k-1) \log \left(-(\log \epsilon)^{-1}\right)=\log \operatorname{det} \hat{B}^{-1}-(k-1) \log \log (1 / \epsilon)$.

Next, we compute $\log \operatorname{det} \hat{B}$. For each $i$ between 1 and $k-1$, we let $g_{i}=f_{0, i}-f_{0, k}$. Then we have

$$
\hat{B} g_{i}=\Pi_{\mathcal{C}}\left(b_{i} f_{0, i}-b_{k} f_{0, k}\right)=b_{i} g_{i}-\frac{b_{i}-b_{k}}{k} \sum_{j=i}^{k} g_{j} .
$$

So we must take the log of the determinant of a matrix whose $(i, j)$ entry is

$$
\frac{b_{k}-b_{j}}{k}+b_{j} \delta_{i j}
$$

The determinant must be a polynomial in the $\left\{b_{i}\right\}$ of joint degree $k-1$. Since the numbering was arbitrary, it must be symmetric. Moreover, by inspection, it has 
degree at most 1 in each of the $b_{i}$ between 1 and $k-1$, and hence by symmetry also in $b_{k}$. These observations force the polynomial to be $\beta$ times a constant which depends only on $k$. But if each $b_{i}=1$, then $\hat{B}=I d$ and the determinant is 1 ; therefore, the constant is 1 and $\log \operatorname{det} \hat{B}=\log \beta$. We have now shown that

$$
\log \operatorname{det} A_{\epsilon}=-(k-1) \log \log (1 / \epsilon)+\sum_{i=1}^{k} \log \pi b_{i} \alpha_{i}-\log \beta .
$$

Finally, we need to go from $\log \operatorname{det} \epsilon R_{\epsilon}$ to $\log \operatorname{det} R_{\epsilon}$. The argument is similar to Proposition 2.3. since the eigenvalues scale by $\epsilon$ in this case, as opposed to $\epsilon^{-2}$, one can easily compute

$$
\log \operatorname{det} \epsilon R_{\epsilon}=\log \operatorname{det} R_{\epsilon}+\zeta_{R_{\epsilon}}(0) \log \epsilon .
$$

We must still compute $\zeta_{R_{\epsilon}}(0)$, but it turns out to be simple:

Proposition 3.6. For any $\epsilon, \zeta_{R_{\epsilon}}(0)=-1$.

Proof. Fix $\epsilon$. The result follows by using homogeneity; we examine (2.1) and note that the same gluing formula applies to $\left(M, \delta^{2} g\right)$ instead of $(M, g)$, for any $\delta$. We then compute the variation of each of the terms. For each of the $\log \operatorname{det} \Delta$ terms, we may apply Proposition 2.3. The volume term $\log V$ changes by $2 \log \delta$, and the boundary length term $\log \left(l\left(\partial M_{\epsilon}\right)\right)$ changes by $\log \delta$. Since the eigenvalues of $R_{\epsilon}$ scale by $\delta^{-1}, \log \operatorname{det} R_{\epsilon}$ changes by $-\zeta_{R}(0) \log \delta$. Putting everything together, we have

$-2 \zeta_{M}(0) \log \delta=-2 \zeta_{M_{\epsilon}}(0) \log \delta-2 \sum_{i=1}^{k} \zeta_{C_{\alpha_{i}, b_{i} \epsilon}}(0) \log \delta+2 \log \delta-\log \delta-\zeta_{R_{\epsilon}}(0) \log \delta$.

Since $\delta$ is arbitrary, we see that

$$
\zeta_{R_{\epsilon}}(0)=2 \zeta_{M}(0)-2 \zeta_{M_{\epsilon}}(0)-2 \sum_{i=1}^{k} \zeta_{C_{\alpha_{i}, b_{i} \epsilon}}(0)-1 .
$$

Recall that for any surface $\Omega$ with or without boundary, $\zeta_{\Omega}(0)=\frac{1}{6} \chi(\Omega)$ Ros]. When $\Omega$ has conical singularities, work of Cheeger Ch2 shows that the same formula holds, with an additional contribution from each conic singularity depending only on the cone angle $\alpha$. Applying these formulas to (3.12), we see that all the contributions from the conic singularities cancel, and we are left with

$$
\zeta_{R_{\epsilon}}(0)=\frac{1}{3}\left(\chi(M)-\chi\left(M_{\epsilon}\right)-\sum_{i=1}^{k} \chi\left(C_{\alpha_{i}, b_{i} \epsilon}\right)\right)-1 .
$$

However, $\chi(M)=\chi\left(M_{\epsilon}\right)+\sum_{i=1}^{k} \chi\left(C_{\alpha_{i}, b_{i} \epsilon}\right)$ by the definition of the Euler characteristic, and hence $\zeta_{R_{\epsilon}}(0)=-1$.

Combining this proposition with (3.10), (3.11), and Lemma 3.5 completes the proof of Theorem 3.1, and thus also finishes the proof of Theorems 1.1 and 1.2 ,

\subsection{Proof of Lemma 3.5.}

Proof. The proof is based on similar arguments in Lee, We. Since $\epsilon R_{\epsilon}=A_{\epsilon}+K_{\epsilon}$, we have formally that

$\log \operatorname{det} \epsilon R_{\epsilon}-\log \operatorname{det} A_{\epsilon}=\int_{0}^{1} \frac{d}{d t} \log \operatorname{det}\left(A_{\epsilon}+t K_{\epsilon}\right) d t=\int_{0}^{1} \operatorname{Tr}\left(\left(A_{\epsilon}+t K_{\epsilon}\right)^{-1} K_{\epsilon}\right) d t$, 
and hence, whenever $\left\|K_{\epsilon} A_{\epsilon}^{-1}\right\|$ has norm less than 1 ,

$$
\begin{gathered}
\left|\log \operatorname{det} \epsilon R_{\epsilon}-\log \operatorname{det} A_{\epsilon}\right| \leq \int_{0}^{1}\left\|A_{\epsilon}^{-1}\right\| \cdot\left\|\left(I d+t K_{\epsilon} A_{\epsilon}^{-1}\right)^{-1}\right\| \cdot \operatorname{Tr}\left|K_{\epsilon}\right| d t \\
\leq \int_{0}^{1}\left\|A_{\epsilon}^{-1}\right\| \cdot \frac{1}{1-\left\|t K_{\epsilon} A_{\epsilon}^{-1}\right\|} \cdot \operatorname{Tr}\left|K_{\epsilon}\right| d t \\
\leq\left\|A_{\epsilon}^{-1}\right\| \cdot \frac{1}{1-\left\|K_{\epsilon} A_{\epsilon}^{-1}\right\|} \cdot \operatorname{Tr}\left|K_{\epsilon}\right| .
\end{gathered}
$$

In order for this calculation to be justified, $K_{\epsilon}$ must be trace class and $\left\|K_{\epsilon} A_{\epsilon}^{-1}\right\|$ must have norm less than 1. As we analyze (3.13), we will prove that both of these conditions hold for sufficiently small $\epsilon$. The first step in that analysis is an observation which is an immediate consequence of the definition of $A_{\epsilon}$ :

Proposition 3.7. $A_{\epsilon}$ is invertible, and the norm of the inverse is bounded by $\left\|B^{-1}\right\| \log (1 / \epsilon)$ for sufficiently small $\epsilon$. On the other hand, if $k=1$ and $b_{1}=1$, then $A_{\epsilon}=-2|\mathcal{V}|$ and hence $\left\|A_{\epsilon}^{-1}\right\|=\alpha_{1} / 2$.

Next, we show that $K_{\epsilon}$ is trace class and estimate its trace norm.

Proposition 3.8. $K_{\epsilon}$ is trace class for all $\epsilon \in(0,1)$, and

$$
\lim _{\epsilon \rightarrow 0} \log (1 / \epsilon) \operatorname{Tr}\left|K_{\epsilon}\right|=0 .
$$

Moreover, if $k=1, b_{1}=1$, and $\epsilon \leq 2^{-\alpha_{1}}$,

$$
\operatorname{Tr}\left|K_{\epsilon}\right| \leq \frac{12}{\alpha_{1}} \epsilon^{1 / \alpha_{1}}
$$

Proof. Since $B$ and $B^{-1}$ both have bounded norm, it suffices to analyze $B K_{\epsilon}$ instead. We compute the trace directly. First analyze the trace of $B K_{\epsilon}$ on $L_{0}^{2}$. As in We, the operator $|\mathcal{V}|+\mathcal{V}\left(\mathcal{T}_{\epsilon}\right)^{-1}$ is diagonal on $L_{0}^{2}$, with eigenvalues

$$
\frac{m}{\alpha_{j}} \frac{\epsilon^{m / \alpha_{j}}+\epsilon^{-m / \alpha_{j}}}{\epsilon^{m / \alpha_{j}}-\epsilon^{-m / \alpha_{j}}}+\frac{|m|}{\alpha_{j}}=\frac{2|m|}{\alpha_{j}} \frac{1}{1-\epsilon^{-2|m| / \alpha_{j}}}
$$

for each $j$ between 1 and $k$. The sum over $m$ and $j$ of the absolute value of these eigenvalues converges for all $\epsilon<1$. Moreover, let $\alpha=\min _{j=1}^{k}\left\{\alpha_{j}\right\}$; this sum times $\epsilon^{-1 / \alpha}$ is bounded by

$$
\sum_{m=1}^{\infty} \sum_{j=1}^{k} \frac{4 m}{\alpha_{j}} \epsilon^{\frac{2 m-1}{a_{j}}} .
$$

On the other hand, the operator $\mathcal{V}\left(\mathcal{U}_{\epsilon}^{-}\right)^{-1}$ is diagonal on $L_{0}^{2}$ with eigenvalues

$$
\frac{m}{\alpha_{j}\left(\epsilon^{m / \alpha_{j}}-\epsilon^{-m / \alpha_{j}}\right)} ;
$$

the sum of the absolute values again converges for all $\epsilon<1$. After multiplying by $\epsilon^{-1 / \alpha}$, it is bounded by

$$
\sum_{m=1}^{\infty} \sum_{j=1}^{k} \frac{2 m}{a_{j}} \epsilon^{\frac{m-1}{a_{j}}}
$$


Since $\left\|L_{\epsilon}\right\| \leq 1$, we conclude that $\epsilon^{-1 / \alpha}$ times the trace of $\left|K_{\epsilon}\right|$ on $L_{0}^{2}$ is bounded by

$$
\sum_{m=1}^{\infty} \sum_{j=1}^{k}\left(\frac{4 m}{\alpha_{j}} \epsilon^{\frac{2 m-1}{a_{j}}}+\frac{2 m}{a_{j}} \epsilon^{\frac{m-1}{a_{j}}}\right) \leq \frac{6 k}{\alpha} \sum_{m=1}^{\infty} m \epsilon^{\frac{m-1}{a_{j}}} .
$$

When $\epsilon^{1 / \alpha}<1 / 2$, this is bounded by $12 k / \alpha$. Since $\mathcal{C}=\emptyset$ when $k=1$, the second claim in Proposition 3.8 follows immediately, and we also see that $\log (1 / \epsilon)$ times the trace of $\left|K_{\epsilon}\right|$ on $L_{0}^{2}$ approaches zero in general.

It remains to analyze $B K_{\epsilon}$ on $\mathcal{C}$; we analyze

$$
\log (1 / \epsilon) \Pi_{\mathcal{C}} B K_{\epsilon} \Pi_{\mathcal{C}}=\Pi_{\mathcal{C}} L_{\epsilon} \Pi_{\mathcal{C}} .
$$

The operator (3.16) is finite-dimensional and hence trace class, and the trace norm is bounded by $(k-1)\left\|\Pi_{\mathcal{C}} L_{\epsilon} \Pi_{\mathcal{C}}\right\|$. It suffices to show that $\left\|\Pi_{\mathcal{C}} L_{\epsilon} \Pi_{\mathcal{C}}\right\|$ goes to zero as $\epsilon$ goes to zero. Suppose not. Then there is a sequence $g_{i}$ of functions in $\mathcal{C}$, of norm one in $L^{2}\left(\oplus_{i=1}^{k} S^{1}\right)$, and a sequence $\epsilon_{i} \rightarrow 0$ where $\Pi_{\mathcal{C}} L_{\epsilon_{i}} g_{i}$ has $L^{2}$-norm bounded below. For each $i$, let $u_{+, i}$ be the harmonic function on $M_{\epsilon_{i}}$ with boundary data $g_{i}$. Since $g_{i}$ has norm one in $L^{2}\left(\oplus_{i=1}^{k} S^{1}\right)$ and is piecewise constant, the $L^{\infty}$ norm of $g_{i}$ is bounded by $\frac{1}{2 \pi}<1$ for all $i$. By the maximum principle, $u_{+, i}$ is also bounded by 1 for each $i$. Therefore, as $i \rightarrow \infty$, the Arzela-Ascoli theorem implies that a subsequence of $u_{+, i}$ converges uniformly on compact subsets of $M$ away from the conic tip to a limit function $u$. By standard elliptic theory, the limit $u$ is itself harmonic on $M$ and is obviously bounded by 1 (and therefore is in the Friedrichs domain at each conic point). Therefore, $u$ itself is constant, so $h=\left.u\right|_{\partial M_{1}}$ is globally constant. However, by construction, passing to the ArzelaAscoli subsequence, $L_{\epsilon_{i}} g_{i} \rightarrow h$ uniformly as $i \rightarrow \infty$. So $\Pi_{\mathcal{C}} L_{\epsilon_{i}} g_{i} \rightarrow \Pi_{\mathcal{C}} h$ uniformly (and hence strongly in $L^{2}$ ), but $\Pi_{\mathcal{C}} h=0$. This is a contradiction which completes the proof of the proposition.

Finally, we prove the necessary bounds for $\left\|K_{\epsilon} A_{\epsilon}^{-1}\right\|$.

Proposition 3.9. The norm $\left\|K_{\epsilon} A_{\epsilon}^{-1}\right\|$ approaches zero as $\epsilon \rightarrow 0$. Moreover, when $k=1, b_{1}=1$, and $\epsilon \leq 2^{-\alpha_{1}},\left\|K_{\epsilon} A_{\epsilon}^{-1}\right\| \leq 1 / 2$.

Proof. To prove the first claim, observe from the definition of $A_{\epsilon}$ and $K_{\epsilon}$ that

$$
\begin{aligned}
B K_{\epsilon} A_{\epsilon}^{-1} B^{-1}= & -\frac{1}{2} \Pi_{0}\left(|\mathcal{V}|+\mathcal{V}\left(\mathcal{T}_{\epsilon}\right)^{-1}-\mathcal{V}\left(\mathcal{U}_{\epsilon}^{-}\right)^{-1} L_{\epsilon}\right)|\mathcal{V}|^{-1} \Pi_{0}-\frac{1}{2} \Pi_{\mathcal{C}}\left(\frac{1}{\log (1 / \epsilon)} L_{\epsilon}\right)|\mathcal{V}|^{-1} \Pi_{0} \\
& +\log (1 / \epsilon) \Pi_{0}\left(-\mathcal{V}\left(\mathcal{U}_{\epsilon}^{-}\right)^{-1} L_{\epsilon}\right) \Pi_{\mathcal{C}}+\Pi_{\mathcal{C}} L_{\epsilon} \Pi_{\mathcal{C}}
\end{aligned}
$$

We analyze each term in (3.17) separately and show that its norm goes to zero as $\epsilon$ goes to zero. For the first term, note that ||$|\mathcal{V}|^{-1} \|$ is bounded by $\alpha=\max _{i}\left\{\alpha_{i}\right\}$ and $\left\|L_{\epsilon}\right\| \leq 1$. The remaining operators are diagonalized by the $f_{m, j}$, and by the eigenvalue calculations in the proof of Proposition 3.8, all the eigenvalues go to zero as $\epsilon$ goes to zero; this is more than sufficient. Similarly, the norm of the second term is bounded by $(\alpha / 2)(\log (1 / \epsilon))^{-1}$, which goes to zero as $\epsilon$ goes to zero. We again use $\left\|L_{\epsilon}\right\|<1$ and the eigenvalue calculations from the previous proposition to analyze the third term; the eigenvalues of $\mathcal{V}\left(\mathcal{U}_{\epsilon}^{-}\right)^{-1}$ go to zero even when multiplied by $\log (1 / \epsilon)$, which gives the norm bound. Finally, we have already done the necessary analysis for the fourth term in the proof of Proposition 3.8. This completes the proof of the claim and hence of the general case of the proposition. 
As for the $k=1$ and $b_{1}=1$ bound, in this case, $A_{\epsilon}^{-1}$ is given by multiplication by $-\frac{\alpha_{1}}{2|m|}$ on each mode. Since $\left\|L_{\epsilon}\right\| \leq 1$, the norm $\left\|K_{\epsilon} A_{\epsilon}^{-1}\right\|$ is bounded by the maximum of $\frac{\alpha_{1}}{2|m|}$ times (3.14) plus the maximum of $\frac{\alpha_{1}}{2|m|}$ times (3.15). Using a similar analysis as in the proof of Proposition 3.8 we see that this is at most

$$
\epsilon^{2 / \alpha_{j}}+\frac{1}{2} \epsilon^{1 / \alpha_{j}}
$$

which is certainly bounded by $1 / 2$ when $\epsilon \leq 2^{-\alpha_{j}}$ (a sharp bound is not necessary). This completes the proof.

It is now an immediate consequence of the three propositions that (3.13) approaches zero as $\epsilon \rightarrow 0$. Moreover, in the $k=1, b_{1}=1, \epsilon \leq 2^{-\alpha_{1}}$ case, we see that (3.13) is bounded by $12 \epsilon^{1 / \alpha_{1}}$. This completes the proof of Lemma 3.5

\section{ACKNOWLEDGEMENTS}

This paper is a generalization of the final chapter of the author's Ph.D. thesis at Stanford [Sh. The author is deeply grateful to his advisor, Rafe Mazzeo, for all his help and support. Additionally, this paper would not have been written without a discussion with Richard Wentworth; the author would like to thank him and also the other organizers of the conference 'Analysis, Geometry, and Surfaces' at Autrans in March 2011. Finally, the author would like to thank Alexey Kokotov, Andras Vasy, and the anonymous referee for helpful comments and bug-spotting, as well as the ARCS foundation for support in 2011-2012.

\section{REFERENCES}

[BFK] D. Burghelea, L. Friedlander, and T. Kappeler, Meyer-Vietoris type formula for determinants of elliptic differential operators, J. Funct. Anal. 107 (1992), no. 1, 34-65, DOI 10.1016/0022-1236(92)90099-5. MR.1165865 (93f:58242)

[Ch2] Jeff Cheeger, Spectral geometry of singular Riemannian spaces, J. Differential Geom. 18 (1983), no. 4, 575-657 (1984). MR730920 (85d:58083)

[GKM] Juan B. Gil, Thomas Krainer, and Gerardo A. Mendoza, Resolvents of elliptic cone operators, J. Funct. Anal. 241 (2006), no. 1, 1-55, DOI 10.1016/j.jfa.2006.07.010. MR2264246 (2007j:58032)

[Kh] Hala Halim Khuri, Determinants of Laplacians on the moduli space of Riemannian surfaces, Thesis (Ph.D.)-Stanford University, 1990, ProQuest LLC, Ann Arbor, MI. MR2638449

[Kh2] Hala Halim Khuri, Heights on the moduli space of Riemann surfaces with circle boundaries, Duke Math. J. 64 (1991), no. 3, 555-570, DOI 10.1215/S0012-7094-91-06427-6. MR:1141285 (93c:58227)

[Kim] Young-Heon Kim, Surfaces with boundary: their uniformizations, determinants of Laplacians, and isospectrality, Duke Math. J. 144 (2008), no. 1, 73-107, DOI 10.1215/001270942008-032. MR2429322(2009f:58052)

[Ko] A. Kokotov, On the asymptotics of determinant of Laplacian at the principal boundary of the principal stratum of the moduli space of Abelian differentials, Trans. Amer. Math. Soc. 364 (2012), no. 11, 5645-5671, DOI 10.1090/S0002-9947-2012-05695-9. MR2946925

[Lee] Yoonweon Lee, Burghelea-Friedlander-Kappeler's gluing formula for the zeta-determinant and its applications to the adiabatic decompositions of the zeta-determinant and the analytic torsion, Trans. Amer. Math. Soc. 355 (2003), no. 10, 4093-4110 (electronic), DOI 10.1090/S0002-9947-03-03249-5. MR.1990576 (2004e:58058)

[LMP] Paul Loya, Patrick McDonald, and Jinsung Park, Zeta regularized determinants for conic manifolds, J. Funct. Anal. 242 (2007), no. 1, 195-229, DOI 10.1016/j.jfa.2006.04.014. MR2274020 (2007g:58036)

[MS] H. P. McKean Jr. and I. M. Singer, Curvature and the eigenvalues of the Laplacian, J. Differential Geometry 1 (1967), no. 1, 43-69. MR0217739 (36 \#828) 
[Mo] Edith Mooers, The heat kernel for manifolds with conic singularities, Thesis (Ph.D.)Massachusetts Institute of Technology, 1996, ProQuest LLC, Ann Arbor, MI. MR2716652

[OPS1] B. Osgood, R. Phillips, and P. Sarnak, Extremals of determinants of Laplacians, J. Funct. Anal. 80 (1988), no. 1, 148-211, DOI 10.1016/0022-1236(88)90070-5. MR960228 (90d:58159)

[OPS2] B. Osgood, R. Phillips, and P. Sarnak, Compact isospectral sets of surfaces, J. Funct. Anal. 80 (1988), no. 1, 212-234, DOI 10.1016/0022-1236(88)90071-7. MR960229 (90d:58160)

[OPS3] B. Osgood, R. Phillips, and P. Sarnak, Moduli space, heights and isospectral sets of plane domains, Ann. of Math. (2) 129 (1989), no. 2, 293-362, DOI 10.2307/1971449. MR986795 (91a:58196)

[Ros] Steven Rosenberg, The Laplacian on a Riemannian manifold, An introduction to analysis on manifolds, London Mathematical Society Student Texts, vol. 31, Cambridge University Press, Cambridge, 1997. An introduction to analysis on manifolds. MR,1462892 (98k:58206)

[Sh] D.A. Sher, Conic degeneration and the determinant of the Laplacian. Ph.D. thesis, Stanford University, 2012.

[Sp] M. Spreafico, Zeta function and regularized determinant on a disc and on a cone, J. Geom. Phys. 54 (2005), no. 3, 355-371, DOI 10.1016/j.geomphys.2004.10.005. MR2139088 (2005k:11184)

[We] Richard A. Wentworth, Precise constants in bosonization formulas on Riemann surfaces. I, Comm. Math. Phys. 282 (2008), no. 2, 339-355, DOI 10.1007/s00220-008-0560-z. MR2421480(2009f:58054)

Department of Mathematics and Statistics, McGill University, 805 Sherbrooke Street West, Montréal, Quebec H3A 2K6, Canada - And - Centre de Recherches Mathématiques, Université de Montréal CP 6128 sucC Centre-Ville, Montréal, H3C 3J7, CANADA

Current address: Department of Mathematics, University of Michigan, 2074 East Hall, 530 Church Street, Ann Arbor, Michigan 48109-1043

E-mail address: dsher@umich.edu 